\title{
Reliability of PEA Measurement in Presence of an Air Void Defect
}

\author{
Antonino Imburgia ${ }^{1}\left(\mathbb{D}\right.$, Pietro Romano ${ }^{1, *(\mathbb{D}}$, Giuseppe Rizzo ${ }^{1}\left(\mathbb{D}\right.$, , Fabio Viola ${ }^{1}\left(\mathbb{D}\right.$, Guido Ala ${ }^{1} \mathbb{D}$ \\ and George Chen ${ }^{2}$ \\ 1 L.E.PR.E. HV Laboratory, Dipartimento di Ingegneria, Università di Palermo, Viale delle Scienze, Edificio 9, \\ 90128 Palermo, Italy; antonino.imburgia01@unipa.it (A.I.); giuseppe.rizzo07@unipa.it (G.R.); \\ fabio.viola@unipa.it (F.V.); guido.ala@unipa.it (G.A.) \\ 2 The Tony Davies High Voltage Laboratory, University of Southampton, Southampton SO17 1BJ, UK; \\ gc@ecs.soton.ac.uk \\ * Correspondence: pietro.romano@unipa.it
}

Received: 30 September 2020; Accepted: 27 October 2020; Published: 29 October 2020

\begin{abstract}
This paper deals with the reliability of the Pulsed Electro-Acoustic (PEA) technique in the case of a specimen containing an air layer. The first approach to this study has been proposed by the authors in previously published works. In these papers, the mathematical description, the PEA cell simulation model, and some experimental tests have been reported. In this work, a more accurate description of the acoustic wave behavior within the PEA cell and specimen with and without an air layer is given. Moreover, the comparison between simulation and experimental tests for both cases (specimen with and without air layer) allowed the validation of the previously developed PEA cell simulation model. The latter was previously validated only for a single layer specimen, here the good performances of the model have also been confirmed in the case of a multilayer specimen, also with an air layer. Experimental and simulation results show that the air layer acts as a barrier for the acoustic signal, due to the different acoustic impedance between the air and the solid dielectric material which constitute the specimen. Therefore, the aim of the present work is to demonstrate that in the case of a three-layers specimen, composed as dielectric-air-dielectric, the PEA cell is not able to provide the complete profile of the entire specimen under test.
\end{abstract}

Keywords: space charge; HVDC; air void; PEA method; multilayer

\section{Introduction}

The Pulsed Electro-Acoustic (PEA) is the most used technique for measuring the accumulation of space charge in solid dielectrics [1]. This measurement technique is quite consolidated especially for testing single layer specimens [2]. Nevertheless, during the years, several experimental tests were carried out in specimens composed of multiple layers [3-5]. This was with the aim to analyze the accumulation of space charge in samples simulating the different High Voltage Direct Current (HVDC) components, e.g., cables and joints [6-8].

However, measurements in real components have also been made with different types of PEA cells. Hozumi in [9] measured the space charge in a cable specimen, while Takeda in [10] proposed the measure of space charge in a full-size cable. Further PEA cell types, named 2-D and 3-D PEA, have been developed for testing other kinds of specimens, e.g., the joints [1].

With reference to the space charge measurement in multilayer flat specimens, two- or three-layered samples are typically tested. In these specimens, due to the different acoustic impedances between the multiple layers placed in contact with each other, careful attention must be paid to the reflection 
phenomenon of the acoustic waves [11,12]. If the reflected waves are not taken into account, the obtained space charge profile could be incorrectly interpreted [13].

Based on the reflection phenomenon mentioned above, the authors proposed several works, as well as a MATLAB model simulating the acoustic wave behavior within the PEA cell [13-18].

In the previously published works, authors have shown that each PEA cell (on the basis of its ground electrode and absorber thicknesses) is suitable to measure space charge on dielectrics with sound velocity and thickness in a fixed range of values $[18,19]$. If the sample's features fall outside the range allowed by the adopted PEA cell, a certain amount of reflected signals are present within the main space charge profile. Therefore, the PEA cell output signal results are disturbed and the real charge distribution is difficult to interpret. This aspect has also been recently demonstrated by several studies in $[20,21]$.

As is well known, over the space charge phenomenon, the partial discharge phenomena represent the further main degradation factor for the insulators employed in the field of High Voltage Direct Current (HVDC) systems. Considering that the trigger of the discharge needs the availability of electrons, both of the above-mentioned phenomena are strongly correlated [22-28].

In recent papers, with the aim to simultaneously measure space charge and partial discharge, specimens containing an air layer have been investigated. In particular, the study of the acoustic wave behavior followed by experimental tests has been carried out in specimens with a configuration of dielectric-air-dielectric [13,29].

In these works, authors found that due to the reflection and transmission coefficients of the air layer in contact with a dielectric material, the reflection phenomenon is strongly accentuated.

What was previously obtained has been confirmed in the present paper, where was also found that the air layer acts as a barrier for the acoustic wave.

Based on the above, the aim of this work is to demonstrate that in the case of a specimen containing an air layer, it is not possible to perform the measurement of the space charge with the PEA method. Compared to the previously published works [13,29] where only experimental tests and a brief theoretical description were given, here a detailed study and analysis of the behavior of the wave are provided, as well as simulations and experimental tests for different multilayer specimens configurations, in order to confirm the predicted behavior.

Concerning the PEA cell simulation model, it has already been widely explained and validated for a single layer specimen [14]. In the present work, it is used to confirm the predictable and obtainable experimental behaviors, thus only the simulation results are reported.

In this way, the validation of the developed model in the case of a multilayer specimen has also been carried out.

\section{Mathematical Model}

In Figure 1, the block diagram of the PEA cell with a multilayer specimen composed of three layers is reported.

The generators $H V D C$ and $e_{p}(t)$ are the high voltage and the pulse voltage sources, respectively. The resistor $R$ is inserted for the protection of the HVDC generator, while $C$ is the decoupling capacitor between the two generators. The HVDC generator, applied by means of the HV and ground electrode, is used to promote the charge accumulation, while $e_{p}(t)$ allows the charges vibration. Due to the charges vibration, acoustic waves are generated from each charge.

These waves travel within the PEA cell and reach the sensor which converts the acoustic wave into the electrical signal. The latter has a very low magnitude, thus an amplifier is used to increase its voltage level. The absorber component, which is mechanically protected by the bottom electrode, has the task of absorbing the waves passing through the sensor, with the purpose of attenuating the magnitude of reflected waves [1,2]. 


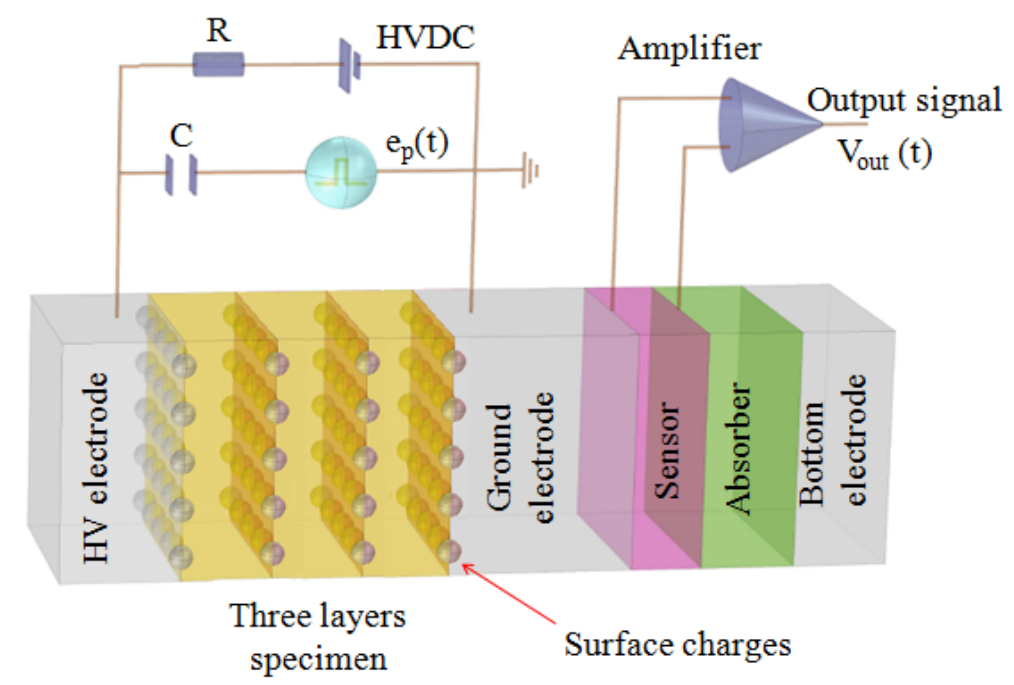

Figure 1. Block diagram of the Pulsed Electro-Acoustic (PEA) cell in the case of a multilayer specimen.

\section{Acoustic Wave Behavior Within the PEA Cell}

The acoustic wave behavior within the PEA cell is characterized by the reflection phenomenon. When an acoustic wave, starting from the deposited charges, reaches an interface, the behavior changes due to the resulting partial transmission and partial reflection of the acoustic wave. This is caused by the mismatch of different acoustic impedance $Z$ values (given by the product between the sound velocity $v$ and density $\rho$ ) of the different materials placed in contact with each other [11].

In each PEA cell components interface, included the specimen, the transmission $K^{T}$, and the reflection $K^{R}$ coefficients are calculated as:

$$
K_{i-j}^{T}=\frac{2 Z_{j}}{Z_{i}+Z_{j}} ; \quad K_{i-j}^{R}=\frac{Z_{j}-Z_{i}}{Z_{i}+Z_{j}} ;
$$

where, the subscript $i$ indicates the material from which the wave is generated or comes from, while the subscript $j$ indicates the material the wave is traveling to.

To evaluate the presence of reflected waves in the main charge profile, the time $t$ (depending on the sound velocity $v$ and thickness $d$ ) needed across each PEA cell component and specimen also has to be taken into account.

The knowledge of the acoustic wave behavior within the PEA cell, as well as inside the specimen under test, is important for the correct interpretation of the obtained space charge profile. Thanks to this preliminary study, the reflected waves can be identified and separated from the main space charge signal. The main charge distribution, considering only the accumulated surface charge in the dielectric interfaces, is composed of only two peaks in the case of a single layer specimen. For two or three layers specimens, instead, the number of main charge peaks is equal to three or four, respectively. If some reflected waves fall inside the main charge profile, further peaks will be present in the PEA cell output signal. This phenomenon occurs for two main causes, such as the PEA cell components dimensions (ground electrode, sensor, and absorber thicknesses) and the specimen features, such as thickness and sound velocity. However, each PEA cell allows us to correctly measure the accumulated charge (without reflected signal) in specimens of dielectric material with features in a specific range of values. To facilitate the choice of sample features based on the used PEA cell, some useful graphs have been reported in a previously published work [18,19].

Moreover, a simulation model of the acoustic wave behavior within the PEA cell has been realized and proposed in [14]. It is able to provide the PEA cell output signal composed of the main charge peaks and, if present, the reflected waves. This simulation model will be used in the present work in order to compare simulation results with experimental tests for the different specimen configurations. 


\section{Measurement Procedure and Specimens Under Test}

As reported in the introduction section, the aim of this work is to show that the PEA method is not suitable to measure space charges in the case of multilayer specimens containing an air void. This is because the air layer acts as a barrier for the acoustic waves and thus some charge signals are not able to be detected by the piezoelectric sensor.

In order to demonstrate this, specimens with and without air layers have been considered. Firstly, the acoustic wave behavior has been analyzed in a three-layer PET-PET-PET specimen. This specimen has been investigated for two main reasons, such as the validation of our PEA cell simulation model for three layers specimens and for the comparison with the specimen having an air void. After that, in order to show the inability of the PEA method to measure space charge in case of a specimen containing an air void, a three-layer PET-AIR-PET specimen has been analyzed.

In the proposed study, the presence and the position of the main charge peaks (such as those due to the charge accumulated in the specimen interfaces) and their reflection have been widely investigated. Therefore, the specimens under test have been subjected to the DC voltage with a magnitude value able to generate only the interfacial charges. The applied DC stress is $13 \mathrm{kV}$ for both specimen configurations, while the magnitude of the pulse voltage is fixed to $500 \mathrm{~V}$.

For each specimen, the mathematical description of the acoustic wave behavior and the obtained PEA cell output signal in both experimental and simulation tests have been reported.

\section{Experimental Test}

The PEA cell adopted in our experimental test is represented by the block diagram reported in Figure 1. For the knowledge of the acoustic wave behavior, some geometrical dimensions and physical features of the PEA cell components need to be taken into account. This information, also for the specimen under test, is reported in Table 1.

Table 1. Geometrical and physical features of the PEA cell component and specimen under test.

\begin{tabular}{cccccc}
\hline & \multicolumn{2}{c}{ Specimen } & \multicolumn{3}{c}{ PEA Cell Component } \\
\cline { 2 - 6 } & Layer A & Layer B & Ground Electrode & Sensor & Absorber \\
\hline Material & PET & Air & Aluminum & PVDF & PVDF \\
\hline Thickness $(\mathbf{m m})$ & 0.11 & 0.11 & 10 & $9 \times 10^{-3}$ & $250 \times 10^{-3}$ \\
\hline $\boldsymbol{v}(\mathbf{m} / \mathbf{s})$ & 2558 & 344 & 6420 & 2260 & 2260 \\
\hline $\boldsymbol{\rho}\left(\mathbf{k g} / \mathbf{m}^{\mathbf{3}}\right)$ & 1380 & 1.225 & 2690 & 1780 & 1780 \\
\hline $\boldsymbol{Z}\left(\mathbf{k g} / \mathbf{m}^{\mathbf{2}} \mathbf{s}\right)$ & $3.5 \times 10^{6}$ & 421.4 & $17 \times 10^{6}$ & $4 \times 10^{6}$ & $4 \times 10^{6}$ \\
\hline Time $(\boldsymbol{\mu s})$ & $\tau_{\mathrm{A}}=0.043$ & $\tau_{\mathrm{B}}=0.31$ & $\tau_{\mathrm{GR}}=1.56$ & $\tau_{\mathrm{SE}}=0.003$ & $\tau_{\mathrm{ABS}}=0.11$ \\
\hline
\end{tabular}

As can be seen, the HV and Bottom electrodes are not inserted in Table 1, this is because the acoustic waves affecting these components do not influence the main PEA cell output signal. This aspect will be more comprehensible in the later paragraphs. For a complete description of the wave behavior, the time $\tau$ needed for the acoustic waves to travel within each PEA cell component and dielectric layer must be taken into account. Therefore, it has also been inserted in the last row of Table 1.

Based on the information above, the description of each acoustic wave, which starts from the different interfaces between two materials placed in contact with each other, can be easily done. In fact, it is only necessary to know the time, indicated with $\tau$ in the table, and the transmission $K^{T}$ and reflection $K^{R}$ coefficients (calculated as in Equation (1)) of each interface.

\subsection{Three Layers Specimen, the PET-PET-PET Configuration}

The three layers specimen is widely employed by researchers to measure the charge accumulation in configurations simulating cables, joints, and terminations. For the partial discharge analysis, instead, an air layer is inserted between two layers of solid dielectric in order to simulate internal discharges. 
In a recent paper published by the authors, as well as in other works present in technical literature, the three layers specimen with an air void defect has been adopted to simultaneously measure the partial discharge and the space charge [29]. In literature, this type of specimen configuration has been inserted in the PEA cell in order to measure the accumulated charge before, during, or after the discharges events $[30,31]$. As reported in the introduction section, the aim of this work is to demonstrate that in the case of an air layer the PEA cell output signal does not represent the real accumulated charge. This can be confirmed for two reasons, such as the barrier effect of the air layer for the acoustic waves and the very low acoustic wave generation coefficient in the dielectric/air interface.

Initially, the acoustic wave behavior was investigated for the three layers PET-PET-PET specimen, without the presence of air void. Despite multilayer specimens made of the same dielectric material not typically being investigated, in our work the results are useful for the comparison with the PET-AIR-PET configuration. In this way, the effect of the air layer on the PEA cell output signal is more understandable.

The scheme of the specimen under test inserted between the electrodes of the PEA cell is shown in Figure 2, while the experimental PEA cell output signal is reported in Figure 3.

\section{HV electrode}

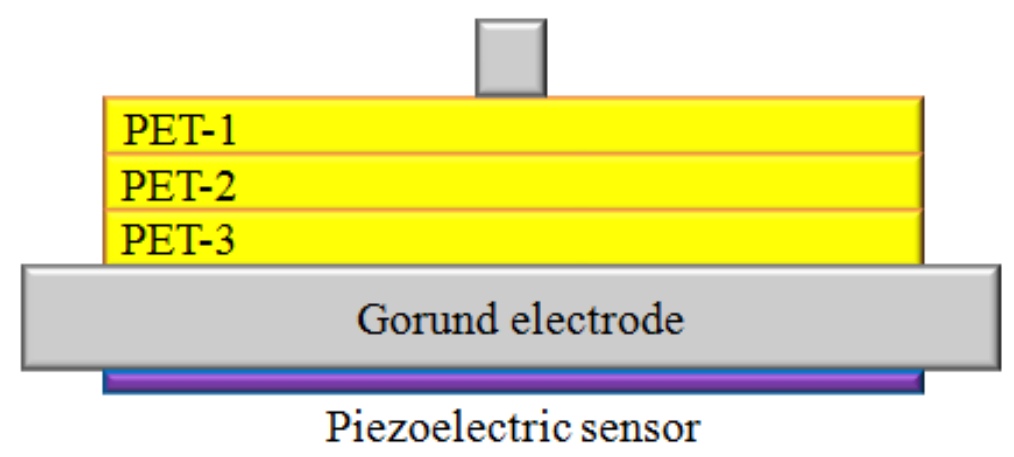

Figure 2. The three layers PET-PET-PET specimen.

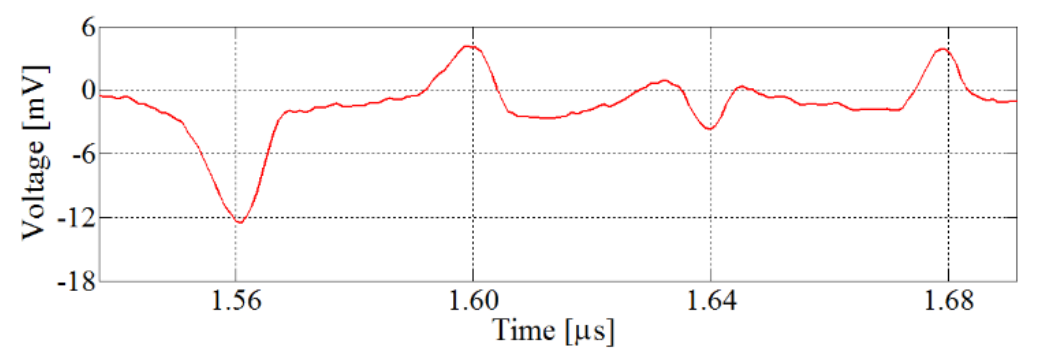

Figure 3. The observed PEA cell output signal for the three layers PET-PET-PET specimen.

As is well known, in the case of a specimen made of the same dielectric material, in the ideal case the interfacial charges should be equal to zero. However, due to the use of silicon oil and the imperfect contact between the dielectric layers, a certain amount of interfacial charge has been observed.

For a better interpretation of the PEA output signal of Figure 3, an analysis of the acoustic wave is needed. First of all, the transmission $K^{T}$ and reflection $K^{R}$ coefficients in each PEA cell components and specimen interfaces have been calculated by means of equation 1 and reported in Figure 4 .

Based on these values, including those reported in Table 1, the acoustic wave behavior within the PEA cell and the specimen can be described as shown in Figure 5.

As can be seen in Figure 4, due to the same dielectric material used for the specimen, the transmission coefficient $K^{T}$ between each PET/PET interface is equal to 1 . This means that the acoustic wave passing through the two layers of PET in contact with each other is totally transmitted. Meanwhile, the reflection coefficient in the same PET/PET interfaces is equal to 0 . 


\begin{tabular}{|c|c|c|c|c|}
\hline Component & \multicolumn{2}{|c|}{$\boldsymbol{K}_{i-j}^{T}$} & \multicolumn{2}{|c|}{$K_{i-j}^{R}$} \\
\hline HV electrode & & & $\bigcup^{-0.66}$ & \\
\hline $\begin{array}{l}\text { Layer A } \\
(\text { PET-1) }\end{array}$ & 0.33 & 1 & $\hat{j}$ & $\overbrace{0.66}$ \\
\hline $\begin{array}{l}\text { Layer A } \\
(\text { PET-2) }\end{array}$ & $\begin{array}{l}\downarrow \\
1\end{array}$ & & $\stackrel{0}{4}$ & \\
\hline $\begin{array}{l}\text { Layer A } \\
\text { (PET-3) }\end{array}$ & $\begin{array}{l}1 \\
1\end{array}$ & $\stackrel{0.33}{4}$ & $\stackrel{0.66}{\mathcal{S}}$ & \\
\hline $\begin{array}{c}\text { Ground } \\
\text { Electrode }\end{array}$ & 1.66 & & $\bigcup^{-0.62}$ & $\overbrace{-0.66}$ \\
\hline Sensor & $\begin{array}{r}\quad 0.37 \\
+\end{array}$ & 1 & & $\overbrace{0.62}$ \\
\hline Absorber & 1 & & $\stackrel{0.62}{\mathcal{S}}$ & $\overbrace{0}$ \\
\hline $\begin{array}{l}\text { Bottom } \\
\text { electrode }\end{array}$ & $\underset{1.62}{\downarrow}$ & & & $\overbrace{-0.62}^{1}$ \\
\hline
\end{tabular}

Figure 4. Transmission and reflection coefficient of the PET-PET-PET specimen and PEA cell.

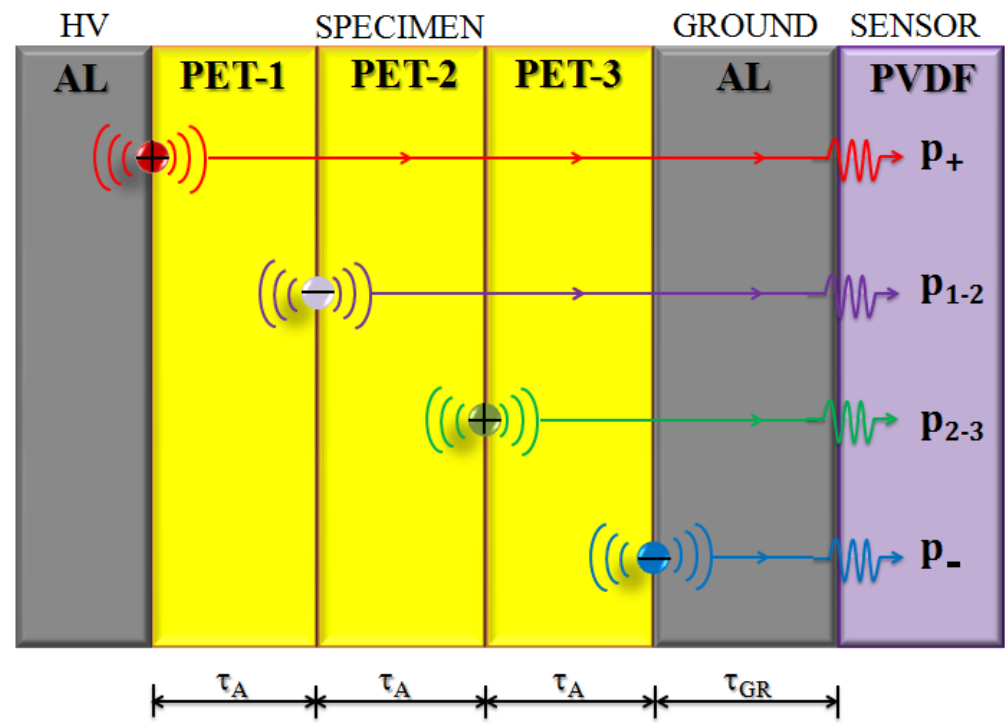

Figure 5. Acoustic wave behavior in the three layers PET-PET-PET specimen.

By observing Figures 3 and 5, the first acoustic wave reaching the sensor is provided by the charges accumulated in the PET-3/Ground electrode interface. In particular, it needs a time equal to $\tau_{G R}=1.56 \mu$ s to reach the sensor, which is the time needed to cross the ground electrode of the PEA cell (see Table 1), while the waves named $p_{2-3}$ and $p_{1-2}$, due to the interfacial charges accumulated in the PET-2/PET-3 and PET-1/PET-2 interfaces, are sensed by the sensor after $\tau_{A}+\tau_{G R}=1.6 \mu \mathrm{s}$ and $\tau_{A}+\tau_{A}+\tau_{G R}=1.64 \mu \mathrm{s}$, respectively. The last acoustic wave, named $p_{+}$, reaches the sensor at $3 \tau_{A}+\tau_{G R}=1.68 \mu \mathrm{s}$.

Based on this study it is possible to confirm that our PEA cell (with its specific components features) is able to correctly measure the space charge in a three layers specimen. The obtained charge distribution and the study of the acoustic wave behavior result are simply to be carried out thanks 
to the specimen made of the same dielectric material. In the case of a layer different from the other two characterizing the three layers specimen, the reflection phenomenon occurs and thus the charge pattern interpretation results more complicated.

In order to investigate and validate, for multilayer specimens, the previously realized PEA cell simulation software [14] has been employed, and in Figure 6 the simulated PEA cell output signal for the specimen configuration, described in Figures 2 and 5, is reported.

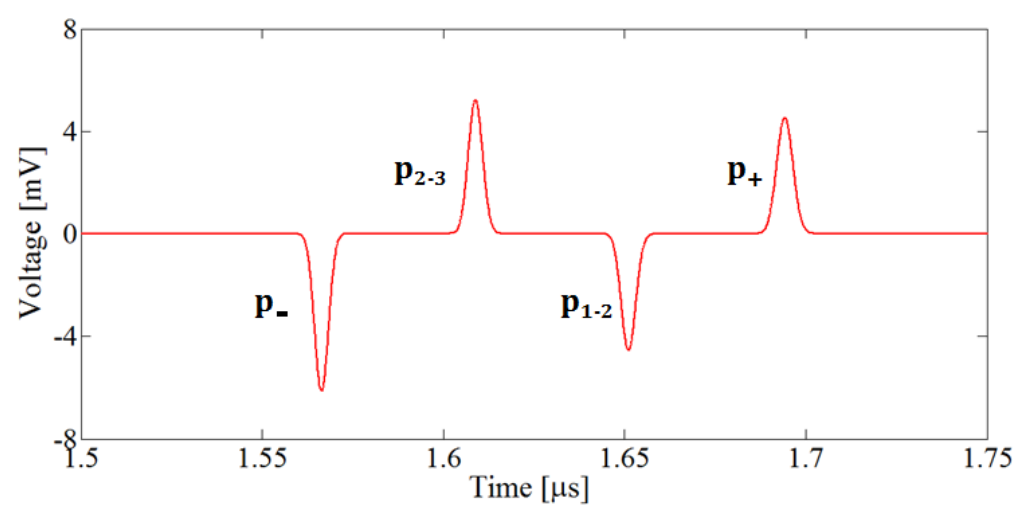

Figure 6. Simulated PEA cell output signal for the PET-PET-PET specimen.

By making a comparison between Figures 3 and 6 (taking also into consideration the waves, named in Figure 5) a good similarity can be observed. In particular, the validation of the model can be confirmed because the polarity and the position of the charge peaks are the same in both patterns.

The differences in the magnitude of the peak do not represent a problem for our aims. However, it could be overcome by knowing the correct attenuation coefficient value of the dielectric material under test. In addition, it is possible to see that in the experimental pattern of Figure 3, the signal between two peaks is different from zero. This is the signal depicted by the oscilloscope, where the deconvolution process has not been applied. On the contrary, in our model, the effect of the RC filter that causes the signal distortion was not implemented, therefore, in the pattern of Figure 6, the signal between two peaks is equal to zero [32].

The distribution of the charge and its sign, found in both experimental and simulation tests, have been validated by making a comparison with the charge profile detected in a multilayer specimen by R. Zou et al. in [33].

\subsection{Three Layers Specimen, the PET-AIR-PET Configuration}

As previously explained, the three-layer specimen made of two layers of solid dielectric and a layer of air interposed between them is a configuration widely investigated by researchers. With reference to the measure of space charge, this specimen configuration has been used to evaluate the effect of charges on the partial discharge, and vice-versa. However, considering the different acoustic impedance of the air layer with respect to other dielectric materials that make up the specimen, the wave reflection phenomenon is strongly present. Therefore, an accurate study of the acoustic wave behavior is essential to avoid an incorrect interpretation of the obtained charge distribution.

Based on the above, in the following three-layer PET-AIR-PET specimen, with configuration reported in Figure 7, has been investigated. 


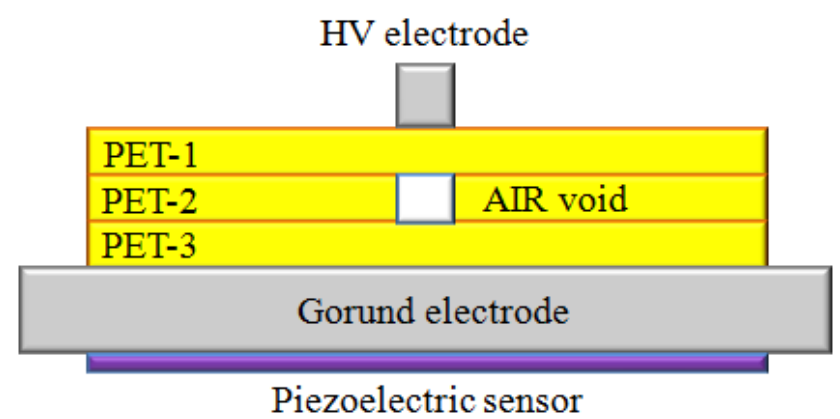

Figure 7. The three layers PET-AIR-PET specimen.

The PEA cell output signal provided by the oscilloscope is shown in Figure 8.

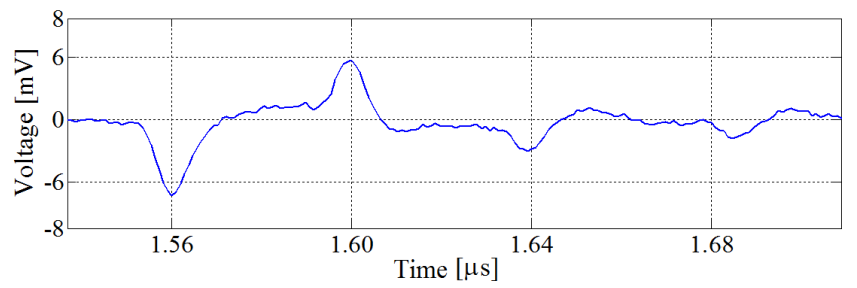

Figure 8. The observed PEA cell output signal for the three layers PET-AIR-PET specimen.

By observing the pattern of Figure 8, seems that the four peaks are associated with the charges accumulated in the four specimen interfaces, as in Figure 5 but with different charge signs. In reality, some peaks are reflected signals that can be easily confused with the accumulated charge. In particular, the first and the second peak are due to the interfacial charge accumulated in the PET/Ground electrode and AIR/PET interfaces, while, the other two peaks are due to the reflection of acoustic waves within the PET layer in contact with the ground electrode. A detailed explanation is given below.

For this specimen, as in the previous case, the transmission and reflection coefficients have been calculated in each interface. For the entire PEA cell, the values of $K^{T}$ and $K^{R}$ are the same as those reported in Figure 4. The same is also the values of $K^{T}$ and $K^{R}$, calculated in the HV/PET and PET/Ground electrode interfaces. In the PET/AIR and AIR/PET interface, instead, the values are different from the previous case and they are reported in Figure 9.

\begin{tabular}{|c|c|c|c|c|}
\hline Component & \multicolumn{2}{|c|}{$\boldsymbol{K}_{i-j}^{T}$} & \multicolumn{2}{|c|}{$K_{i-j}^{R}$} \\
\hline $\begin{array}{l}\text { Layer A } \\
\text { (PET-1) }\end{array}$ & & 2 & $\mathcal{U}^{-0.99}$ & \\
\hline $\begin{array}{c}\text { Layer B } \\
\text { (AIR) }\end{array}$ & $\underset{2.3 \cdot 10^{-4}}{7}$ & $2.3 \cdot 10^{-4}$ & $\stackrel{0.99}{\mathcal{G}}$ & $\underset{0.99}{\bigcap}$ \\
\hline $\begin{array}{l}\text { Layer A } \\
\text { (PET-3) }\end{array}$ & & & & $\underset{-0.99}{\bigcap}$ \\
\hline
\end{tabular}

Figure 9. Transmission and reflection coefficient of the PET-AIR-PET specimen.

The analysis of the values reported in Figure 9 confirms that the air layer acts as a barrier for the acoustic waves. In particular, in the PET-1/AIR interface, $K^{T}$ is equal to $2.3 \cdot 10^{-4}$ for the acoustic waves coming from the PET- 1 and directed towards the AIR layer. This means that only $0.023 \%$ of the wave is transmitted in the sensor direction, while, $99 \%$ is reflected in the opposite direction, due to the value of $K^{R}=-0.99$. The negative sign of $K^{R}$ means that when the wave is reflected, it goes back in the opposite direction with respect to the incident wave. 
In the AIR/PET-3 interface, for the wave coming from the AIR layer and directed towards the PET material, $K^{T}=2$ and $K^{R}=0.99$. Therefore, the part of the acoustic wave able to cross this interface is given by $K^{T}-K^{R}=1.01$, such as $101 \%$. This means that the wave is totally transmitted and a bit amplified, thus this interface result is not influential for the wave behavior.

Based on the explanation above, the complete acoustic wave behavior in the PET-AIR-PET specimen can be schematically described as in Figure 10.

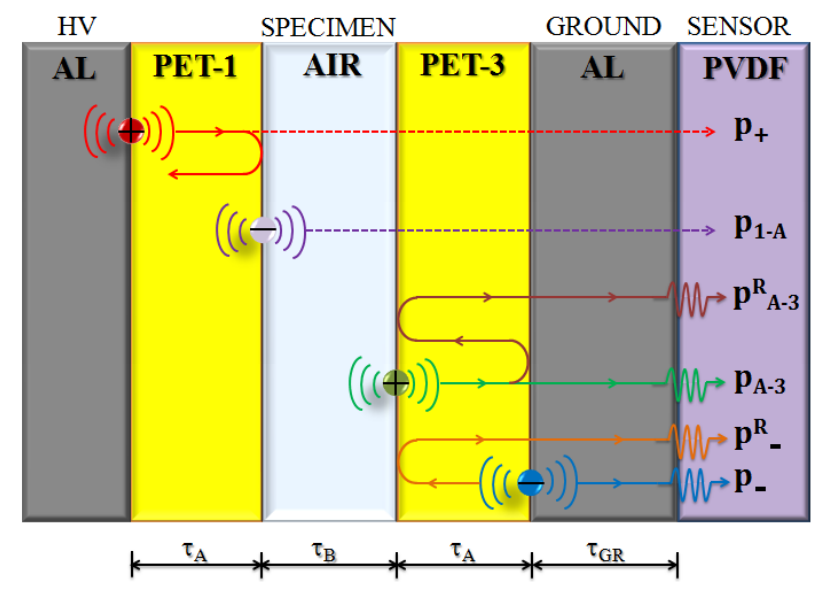

Figure 10. Acoustic wave behavior in the three layers PET-AIR-PET specimen.

By observing Figure 10 and taking into account the time values reported in Table 1, the sequence of the acoustic waves that reach the piezoelectric sensor can be described as follows.

First of all, as in the previous case of Figure 5, the acoustic wave generated in the PET-3/Ground electrode interface, named $p_{-}$, reaches the sensor at a time $\tau=\tau_{G R}=1.56 \mu \mathrm{s}$. After that, the green acoustic wave, which is due to the charge accumulated in the AIR/PET-3 interface, is sensed by the sensor after $\tau=\tau_{A}+\tau_{G R}=1.60 \mu \mathrm{s}$.

Before describing the behavior of the other acoustic waves, it is important to explain the acoustic wave generation. In particular, when a charge is subjected to vibrations, two acoustic waves with opposite polarity are simultaneously generated. If the charge has a positive sign, a positive acoustic wave is generated and directed toward the sensor. Simultaneously, a negative acoustic wave is generated and directed in the opposite direction. On the contrary, if the accumulated charge has a negative sign, the wave directed towards the sensor is negative, while that directed in the opposite direction is positive. For this last case, Figure 11 can be observed.

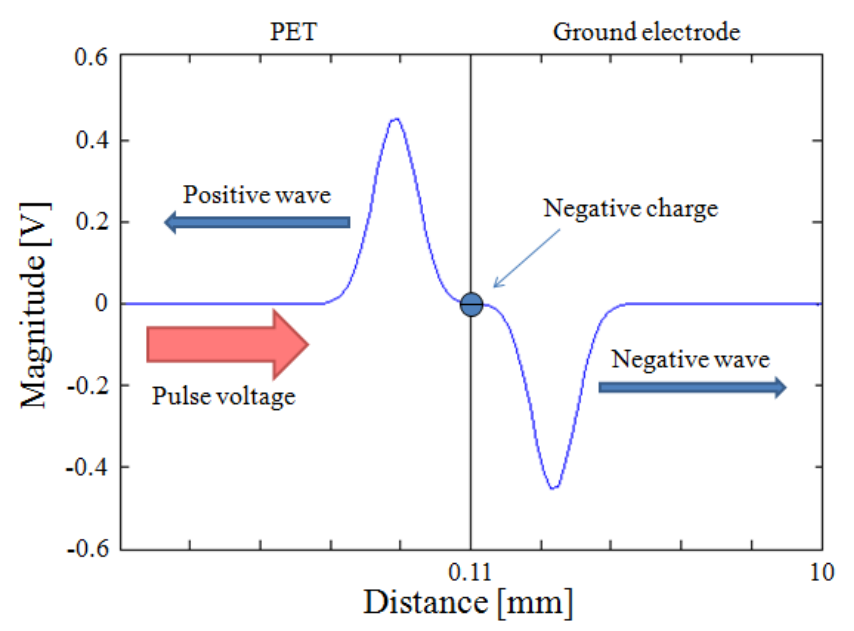

Figure 11. Generation of the acoustic wave in case of negative charge deposited in the PET/Ground electrode interface. 
Based on the above, it is possible to say that the negative interfacial charge deposited in the PET-3/Ground electrode interface generates a positive acoustic wave which travels in the opposite direction. This wave, depicted in orange color, when reaches the AIR/PET-3 interface is for the $99 \%$ reflected in the sensor direction. Moreover, the negative value of $K^{R}=-0.99$ (see Figure 9) means that during the reflection the sign of the wave is also inverted. To better understand what happened in this interface the screenshot of the dynamic simulation provided by our PEA cell model, is shown in Figure 12 [34]. In particular, the simulation has been stopped and printed at time zero (such as the same time instant of Figure 11) and after $0.064 \mu$ s (such as the time needed for the acoustic wave to reach the interface and be reflected).

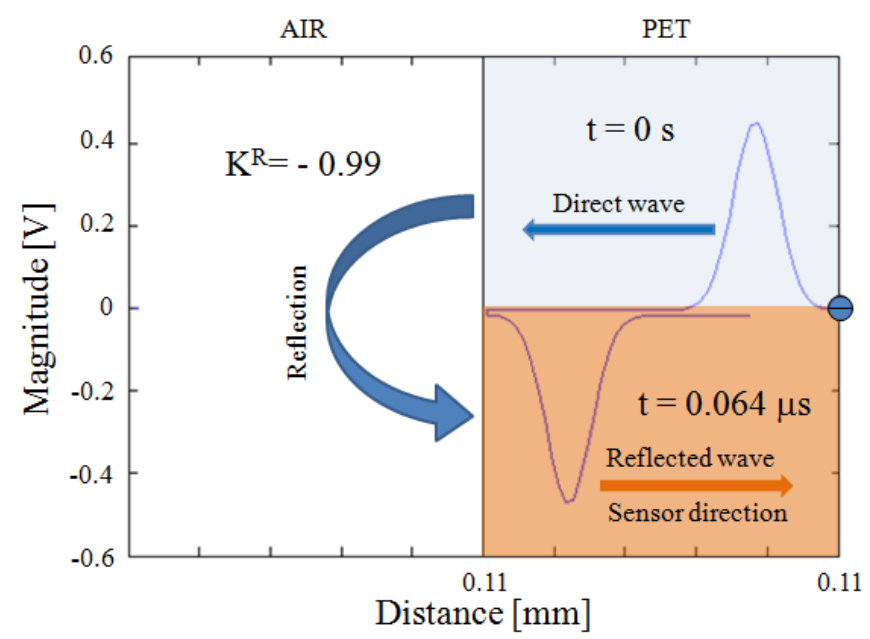

Figure 12. Reflection and sign inversion of the acoustic wave reaching the AIR/PET-2 interface.

However, the orange acoustic wave with negative polarity, named $p_{-}^{R}$, reaches the sensor after $\tau=2 \tau_{A}+\tau_{G R}=1.64 \mu \mathrm{s}$.

Finally, the fourth peak in the pattern of Figure 8 is due to the reflection within the PET-3 layers of the acoustic wave generated by the positive charge deposited in the AIR/PET-3 interface. In Figure 10, this wave is highlighted in the brown color and named $p_{A-3}^{R}$. By observing Figure 10, when the green acoustic wave reaches the PET-3/Ground electrode interface, it is partially transmitted and partially reflected. The reflected part is equal to $66 \%$ (see Figure 4 ) of the incident wave. Therefore, it goes back to maintaining the positive polarity (as the generated wave) and when it reaches the AIR/PET-3 interface is reflected again in the sensor direction. In this interface $K^{R}=-0.99$ and thus, as for the previous orange wave, the polarity of the wave is inverted. In conclusion, after $\tau=3 \tau_{A}+\tau_{G R}=1.68 \mu \mathrm{s}$, the brown acoustic wave, with negative polarity, reaches the sensor. As can be seen, the calculated value $(1.68 \mu \mathrm{s})$ is a bit different from that observed in the oscilloscope, which is around $1.69 \mu \mathrm{s}$ (see Figure 8). This difference can be attributed to the wave propagation delay during the reflection, which is not taken into account in our calculations.

With reference to the acoustic wave generated by the charge deposited in the PET-1/AIR interface, based on the time values reported in Table 1, it should reach the sensor after $\tau=\tau_{B}+\tau_{A}+\tau_{G R}=1.91 \mu \mathrm{s}$. However, we use the dashed (and violet) line because no peaks were observed in that instant of time. This is due to the acoustic wave generation coefficient $K^{G}$, calculated as in equation 2 , which for the PET-1/AIR interface is equal to 0.00012 . However, the generated acoustic wave in this interface has a very low magnitude and thus, due to the attenuation coefficient of the materials involved in the wave path, no signal is sensed by the sensor. On the contrary, in the AIR/PET-3 and PET-3/Ground electrode interface, the values of $K^{G}$ are equal to 0.99 and 0.82 , respectively.

$$
K_{i-j}^{G}=\frac{Z_{j}}{Z_{i}+Z_{j}} ;
$$


Concerning the acoustic wave generated by the charge deposited in the HV electrode/PET-1 interface, the value of $K^{G}$ is equal to $17 \%$ of the incident pulse voltage. Despite this value being low, the generated wave is able to be detected by the sensor. However, as previously widely explained, the barrier effect of the air layer forces the acoustic wave to reflect itself within the PET-1 layer. Therefore, this wave, depicted in the dashed red line, also cannot reach the sensor.

The PET-AIR-PET specimen above described has been implemented in the PEA cell simulation model. The simulated result is reported in Figure 13. The comparison between the simulated and experimental (see Figure 8) profiles confirms the good performances of the developed model for specimens in which an air layer is present. The differences between the simulated and the experimental patterns have been already explained for the previously analyzed PET-PET-PET specimen.

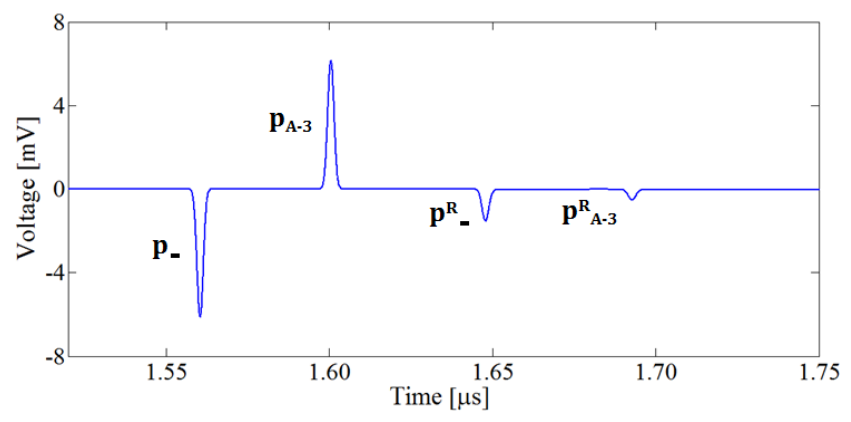

Figure 13. Simulated PEA cell output signal for the PET-AIR-PET specimen.

\section{Results Discussion}

It has been shown by means of the presented study that the PEA technique is not suitable for testing multilayer specimen in which an air layer is present. This is due to the barrier effect of the air material. In particular, the strongly accentuated acoustic wave reflection phenomenon in the air/solid dielectric interfaces is caused by the high difference of the acoustic impedance $Z$. In fact, in our case, $Z_{\text {air }}=421.4 \mathrm{~kg} / \mathrm{m}^{2} \mathrm{~s}$ while $Z_{\mathrm{PET}}=3.5 \times 10^{6} \mathrm{~kg} / \mathrm{m}^{2} \mathrm{~s}$. These values, in turn, generate a high acoustic wave reflection coefficient $K^{R}$ and a very low acoustic wave transmission coefficient $K^{T}$, in the same interface. Therefore, for the acoustic waves traveling within the PEA cell, it is difficult to reach the piezoelectric sensor because they are reflected in the opposite direction. Despite not being demonstrated in this work, the same phenomenon can be also observed when the air layer is placed in contact with the ground electrode of the PEA cell. This is because the acoustic impedance of the aluminum material is much higher than that of air $\left(Z_{\text {aluminum }}=17 \times 10^{6} \mathrm{~kg} / \mathrm{m}^{2} \mathrm{~s}\right)$.

Based on the obtained results, in order to avoid incorrect interpretation of the charge distribution provided by the PEA cell, the authors suggest that the PEA is a preliminary evaluation of the acoustic wave behavior.

\section{Conclusions}

The purpose of this work was to demonstrate that for a specimen containing an air layer, the space charge measurement with the PEA method cannot be correctly made. The motivation that pushed the authors to this study derived from several failed attempts in which the simultaneous detection of the space charge and partial discharges was attempted. In particular, we published some papers in which the charge signal has been detected in a specimen containing an air void. However, after further subsequent studies where the acoustic wave behavior has been widely investigated, it was understood that the previously obtained and published charge profiles were not correctly interpreted. In reality, as reported in the present work, we found that the air layer acts as a barrier for the acoustic wave due to its acoustic impedance values. In the proposed experimental test, in fact, by taking into consideration the generation $K^{G}$, transmission $K^{T}$ and reflection $K^{R}$ coefficients calculated in each specimen interface the following numerical values have been found. In the PET-1/air interface 
$K^{R}=-0.99$ and $K^{T}=2.3 \times 10^{-4}$, this means that the acoustic wave reaching this interface is almost totally reflected in the HV electrode direction. In the AIR/PET-3 interface, instead, $K^{R}=0.99$ and $K^{T}=2$, therefore $K^{T}-K^{R}=1.01$ is the total transmitted wave, which is equal to $101 \%$. This means that in this interface there is no acoustic wave propagation problem. Despite the acoustic wave being able to easily across the AIR/PET-3 interface, no charge peak coming from the PET-1/AIR interface has been observed in the obtained charge distribution due to the low acoustic wave generation coefficient $\left(K^{G}=0.00012\right)$ of the PET-1/AIR interface. However, as a conclusion, it is possible to confirm that the signal displayed by the oscilloscope is only due to the acoustic waves and their reflections generated by the charge accumulated in the PET-3 layer interfaces.

In summary, the air layer does not allow the charge signal coming from the dielectric layer placed in contact with the upper electrode of the PEA cell to reach the sensor. Moreover, we also found that the acoustic wave generation coefficient in the PET/AIR interface is very low. This means that the waves generated in this interface, due to the attenuation phenomenon, are not able to reach the sensor. Therefore, the only acoustic wave correctly detected and observed by the oscilloscope are those due to the charge accumulated in the layer of PET placed in contact with the ground electrode.

In conclusion, it is essential to highlight the importance of the developed PEA cell model for understanding acoustic wave behavior. In particular, the dynamic simulation, which is able to show as a video of the acoustic wave traveling within the PEA cell, results in a very useful tool. However, in the proposed work, the previously developed PEA cell simulation model has been validated for the three layers specimen with the presence of an air void.

In future work, we will focus on the design of a new PEA cell able to measure the space charge in a multilayer specimen with the presence of an air layer.

Author Contributions: Conceptualization, A.I.; Data curation, A.I.; Formal analysis, A.I. and P.R.; Investigation, A.I. and G.R.; Methodology, A.I.; Resources, P.R.; Supervision, G.A. and G.C.; Writing—original draft, A.I.; Writing-review \& editing, A.I., P.R. and F.V. All authors have read and agreed to the published version of the manuscript.

Funding: This research received no external funding.

Conflicts of Interest: The authors declare no conflict of interest.

\section{References}

1. Imburgia, A.; Miceli, R.; Sanseverino, E.R.; Romano, P.; Viola, F. Review of space charge measurement systems: Acoustic, thermal and optical methods. IEEE Trans. Dielectr. Electr. Insul. 2016, 23, 3126-3142. [CrossRef]

2. Mazzanti, G.; Marzinotto, M. Extruded Cables for High Voltage Direct Current Transmission: Advances in Research and Development; John Wiley \& Sons: Hoboken, NJ, USA, 2013.

3. Zhao, X.; Pu, L.; Liu, J.; Duan, W.; Ju, Z.; Sun, H.; Huang, B.; Hao, M.; Chen, G.; Feng, N. Temperature gradient effect on the space charge behaviour in multilayers of oil and pressboard. IEEE Trans. Dielectr. Electr. Insul. 2019, 26, 1645-1653. [CrossRef]

4. Kawano, S.; Ishikawa, K.; Miyake, H.; Tanaka, Y. Space charge accumulation characteristics in multilayered polyimide films under DC high stress. In Proceedings of the IEEE International Conference on Solid Dielectrics (ICSD), Bologna, Italy, 30 June-4 July 2013; pp. 393-396.

5. Huang, B.; Xu, Z.; Hao, M.; Chen, G. Multilayers oil and oil-impregnated pressboard electric field simulation based on space charge. IEEE Trans. Dielectr. Electr. Insul. 2019, 26, 530-538. [CrossRef]

6. Chen, J.; Gao, Y.M.; Zhu, J.L.; Yu, Q. Space charge dynamics in double-layered Insul. cable under polarity reversal voltage. IEEE Trans. Dielectr. Electr. Insul. 2020, 27, 622-630. [CrossRef]

7. Tohmine, T.; Fujitomi, T.; Miyake, H.; Tanaka, Y.; Ida, Y.; Inoue, Y. Measurement of space charge accumulated in multi-layered samples composed of different insulators used in the joints of DC transmission cables. In Proceedings of the International Symposium on Electrical Insulating Materials (ISEIM), Toyohashi, Japan, 11-15 September 2017; pp. 299-302. 
8. Iguchi, K.; Tohmine, T.; Miyake, H.; Tanaka, Y.; Takada, T.; Ida, Y.; Murata, Y.; Inoue, Y. Space charge accumulation characteristics in double-layered samples simulating DC cable joints. In Proceedings of the 12th International Conference on the Properties and Applications of Dielectric Materials (ICPADM), Xi'an, China, 20-24 May 2018; pp. 119-123.

9. Hozumi, N.; Takeda, T.; Suzuki, H.; Okamoto, T. Space charge behaviorin XLPE cable Insul. under 0.2-1.2 MV/cm dc fields. IEEE Trans. Dielectr. Electr. Insul. 1998, 5, 82-90. [CrossRef]

10. Takeda, T.; Hozumi, N.; Suzuki, H.; Fujii, K.; Terashima, K.; Hara, M.; Mutrata, Y.; Wantanabe, K.; Yoshida, M. Space charge behavior in fullsize $250 \mathrm{kV}$ DC XLPE cables. IEEE Trans. Power Deliv. 1998, 13, 28-39. [CrossRef]

11. Bodega, R.; Morshuis, P.H.F.; Smit, J.J. Space charge measurements on multi-dielectrics by means of the pulsed electroacoustic method. IEEE Trans. Dielectr. Electr. Insul. 2006, 13, 272-281. [CrossRef]

12. Bodega, R.; Morshuis, P.H.F.; Smit, J.J. Electrostatic force distribution in a multi-layer dielectric tested by means of the PEA method. IEEE Int. Conf. Solid Dielectr. 2004, 1, 264-267.

13. Imburgia, A.; Romano, P.; Ala, G.; Rizzo, G.; Giglia, G.; Viola, F. Acoustic Wave Behavior in a Specimen Containing an Air Void Defect. In Proceedings of the IEEE Conference on Electrical Insul. and Dielectric Phenomena (CEIDP), Richland, WA, USA, 20-23 October 2019; pp. 470-473.

14. Imburgia, A.; Romano, P.; Ala, G.; Riva Sanseverino, E.; Giglia, G. The Role of Right Interpretation of Space Charge Distribution for Optimized Design of HVDC Cables. IEEE Trans. Ind. Appl. 2019, 55, 7165-7174. [CrossRef]

15. De Araujo Andrade, M.; Candela, R.; De Rai, L.G.; Franchi Bononi, S.; Imburgia, A.; Riva Sanseverino, E.; Romano, P.; Viola, F. Interpretation of PEA Output Signal in a Multilayer Specimen. In Proceedings of the IEEE Conference on Electrical Insul. and Dielectric Phenomena (CEIDP), Cancun, Mexico, 21-24 October 2018; pp. 101-104.

16. Romano, P.; Imburgia, A. Effect of Acoustic Wave Reflections on Space Charge Measurements with PEA Method. In Proceedings of the IEEE 4th International Forum on Research and Technology for Society and Industry (RTSI), Palermo, Italy, 10-13 September 2018; pp. 1-6.

17. Imburgia, A.; Romano, P.; Chen, G.; Huang, B.; Ala, G.; Riva Sanseverino, E.; Viola, F. The Acoustic Wave Behavior Within the PEA Cell for Space Charge Measurement. In Proceedings of the IEEE Conference on Electrical Insul. and Dielectric Phenomena (CEIDP), Cancun, Mexico, 21-24 October 2018; pp. 275-278.

18. Imburgia, A.; Romano, P.; Chen, G.; Rizzo, G.; Riva Sanseverino, E.; Viola, F.; Ala, G. The Industrial Applicability of PEA Space Charge Measurements, for Performance Optimization of HVDC Power Cables. Energies 2019, 12, 4186. [CrossRef]

19. Imburgia, A.; Romano, P.; Riva, S.E.; Rai, L.D.; Bononi, S.F.; Troia, I. Pulsed Electro-Acoustic Method for specimens and cables employed in HVDC systems: Some feasibility considerations. In Proceedings of the AEIT International Annual Conference, Bari, Italy, 3-5 October 2018; pp. 1-6.

20. Hou, Y.; Huang, B.; Chen, G.; Ye, K.; Zhao, X. The improvement of the Pulsed-Electroacoustic (PEA) system measurement from the acoustic wave transportation perspective. In Proceedings of the 12th International Conference on the Properties and Applications of Dielectric Materials (ICPADM), Xi'an, China, 20-24 May 2018; pp. 1024-1027.

21. Huang, B.; Hao, M.; Xu, Z.; Chen, G.; Tang, C.; Hao, J. Research on thickness ratio and multilayers effect on the oil and paper space charge distribution. In Proceedings of the International Conference on Condition Monitoring and Diagnosis (CMD), Xi'an, China, 25-28 September 2016; pp. 40-43.

22. Montanari, G.C. Bringing an Insul. to failure: The role of space charge. IEEE Trans. Dielectr. Electr. Insul. 2011, 18, 339-364. [CrossRef]

23. Rahimi, M.; Javadinezhad, R.; Vakilian, M. DC partial discharge characteristics for corona, surface and void discharges. In Proceedings of the IEEE 11th International Conference on the Properties and Applications of Dielectric Materials (ICPADM), Sydney, Australia, 19-22 July 2015; pp. 260-263.

24. Morshuis, P.; Cavallini, A.; Fabiani, D.; Montanari, G.C.; Azcarraga, C. Stress conditions in HVDC equipment and routes to in service failure. IEEE Trans. Dielectr. Electr. Insul. 2015, 22, 81-91. [CrossRef]

25. Narita, T.; Mima, M.; Miyake, H.; Tanaka, Y.; Kozako, M.; Hikita, M. Investigation for influence of space charge accumulation in motor winding coating material on partial discharge inception voltage. In Proceedings of the IEEE Conference on Electrical Insul. and Dielectric Phenomena (CEIDP), Richland, WA, USA, 20-23 October 2019; pp. 637-640. 
26. Chen, G.; Zhou, C.; Li, S.; Zhong, L. Space charge and its role in electric breakdown of solid Insul. In Proceedings of the IEEE International Power Modulator and High Voltage Conference (IPMHVC), San Francisco, CA, USA, 6-9 July 2016; pp. 120-127.

27. Francis, A.; Martinez, A.; Thompson, K.; Burke, K.; Zirnheld, J. Space charge accumulation as a contributor to partial discharge activity in dielectric elastomer actuators under high voltage DC. In Proceedings of the IEEE International Power Modulator and High Voltage Conference (IPMHVC), San Francisco, CA, USA, 6-9 July 2016; pp. 330-335.

28. Wei, Y.; Mu, H.; Deng, J.; Zhang, G. Effect of space charge on breakdown characteristics of aged oil-paper Insul. under DC voltage. IEEE Trans. Dielectr. Electr. Insul. 2016, 23, 3143-3150. [CrossRef]

29. Imburgia, A.; Romano, P.; Viola, F.; Madonia, A.; Candela, R.; Troia, I. Space charges and partial discharges simultaneous measurements under DC stress. In Proceedings of the IEEE Conference on Electrical Insul. and Dielectric Phenomena (CEIDP), Toronto, ON, Canada, 16-19 October 2016; pp. 514-517.

30. Cavallini, A.; Ciani, F.; Montanari, G.C. The effect of space charge on phenomenology of partial discharges in Insul. cavities. In Proceedings of the Annual Report Conference on Electrical Insul. and Dielectric Phenomena, Nashville, TN, USA, 16-19 October 2005; pp. 410-413.

31. Kan, T.; Abe, K.; Miyake, H.; Tanaka, Y.; Maeno, T. The Influence of corona discharge on space charge accumulation in polyimide film. In Proceedings of the Annual Report Conference on Electrical Insul. and Dielectric Phenomena, Shenzhen, China, 20-23 October 2013; pp. 258-261.

32. Vissouvanadin, B.; Laurent, C.; Le Roy, S.; Teyssèdre, G.; Denizet, I.; Mammeri, M.; Poisson, B. A deconvolution technique for space charge recovery in lossy and dispersive dielectrics using PEA method. In Proceedings of the Annual Report Conference on Electrical Insul. and Dielectic Phenomena, West Lafayette, IN, USA, 17-20 October 2010; pp. 1-4.

33. Zou, R.; Hao, J.; Liao, R. Space/Interface Charge Analysis of the Multi-Layer Oil Gap and Oil Impregnated Pressboard Under the Electrical-Thermal Combined Stress. Energies 2019, 12, 1099. [CrossRef]

34. Imburgia, A. Modelling of Pulsed Electro Acoustic Method for Space Charge Detection on Single and Multilayer Dielectrics. Ph.D. Thesis, University of Palermo, Palermo, Italy, 2018.

Publisher's Note: MDPI stays neutral with regard to jurisdictional claims in published maps and institutional affiliations.

(C) 2020 by the authors. Licensee MDPI, Basel, Switzerland. This article is an open access article distributed under the terms and conditions of the Creative Commons Attribution (CC BY) license (http://creativecommons.org/licenses/by/4.0/). 